\title{
Identidade sexual na infância: crenças, processo evolutivo e fatores associados
}

\section{Sexual identity in childhood: beliefs, evolutionary process and associated factors}

\author{
*Filomena Velho, *Elisabete Brito, *Florbela Rodrigues \\ Instituto Politécnico da Guarda
}

\begin{abstract}
Resumo
Pretendemos conhecer o processo evolutivo e as características das crenças infantis (3-10 anos) acerca de identidade sexual e fatores associados. As variáveis consideradas foram: conservação da identidade sexual verbalizada e conservação da identidade sexual em figuras com contradição (constância sexual). É um estudo empírico onde analisamos a associação das crenças nos domínios considerados, com aspetos sócio demográficos e de desenvolvimento. A amostra é incidental, constituída por 566 crianças tendo sido realizado tratamento estatístico. Com este estudo comprovámos a existência de processos evolutivos ou de diferenciação nas crenças infantis analisadas, associados a vários fatores.

Palavras-chave: identidade sexual, crenças infantis, fatores associados
\end{abstract}

\begin{abstract}
We intend to know the evolutionary process and the characteristics of children's beliefs (3-10 years) about sexual identity and the factors associated. The variables considered were: preservation of verbalized sexual identity and conservation of sexual identity in figures with contradiction (sexual constancy). Is an empirical study where analyze the association of beliefs in the considered domains, with socio-demographic and developmental aspects. The sample is incidental, consisting of 566 children and statistical treatment was performed. We verified the existence of evolutionary processes of differentiation in the children's beliefs analyzed, associated to several factors.
\end{abstract}

Keywords: sexual identity, child beliefs, associated factors

A construção da identidade sexual (como me vejo, como me valoro, como me sinto como pessoa sexuada) é um dos componentes básicos da identidade porque somos bio psico socialmente sexuados (Lopez e Fuertes, 1998). É um processo lento, gradual e complexo que supõe a classificação sexual constante e generalização a partir de uma série de características definidas cultural e socialmente como masculinas ou femininas. Os papéis de género estão presentes ao longo de toda a vida, mas a identidade sexual não é suscetível de mudar. (Badinter, 1992; Calderone e Johnson, 1983; Lopez, 2005; Lopez e Fuertes, 1989; Marques, 2002). É uma construção não apenas a nível biológico, mas também a nível psicológico e social, resultando de uma série de operações psicológicas que se iniciam na infância (determinante na sua estruturação) e se prolongam até ao final da adolescência (Marques, 2002; Mantilla, 2006). A criança distingue a sua identidade sexual ao diferenciar-se das pessoas do sexo oposto e ao identificar-se com as pessoas do mesmo sexo (Badinter, 1992; Lopez, 2005; Lopez e Fuertes, 1989; Marques, 2002). Este processo ocupa um lugar relevante no desenvolvimento, tendo lugar, aproximadamente, entre os 2 e os 7 anos. Gesell (1956), Kohlberg (1972) e Money e Ehrhardt (1972) sublinham a importância da linguagem neste processo. Entre os 2 e os 3 anos, as crianças auto classificam-se como rapazes ou raparigas, mas não distinguem, de forma clara, entre identidade sexual e papel de género. Para as crianças pequenas, a aparência é muito importante para determinar a identidade. Consideram, inicialmente, que as transformações mágicas são possíveis, não percebendo que a identidade é permanente e não pode ser interrompida por transformações aparentes (Bernstein, 1994). O entendimento de que o sexo de uma pessoa permanece o mesmo apesar das mudanças na idade e aparência é chamado de constância do género (Atkinson et al., 1995). Podemos falar de permanência de identidade sexual quando as crianças sabem que têm uma identidade para sempre, que não depende da sua vontade e que se fundamenta nas características do seu corpo e nas do papel social atribuído. Até aos 7 anos, aproximadamente, este processo não incorpora como elemento de classificação a dimensão genital ou anatómica que dará lugar à constância sexual que nos permitirá falar de identidade sexual (Barragan, 1989, 1991; Lopez, 1984, 2005). Só quando as crianças fizerem depender a identidade, das diferenças anatómicas básicas, que é o verdadeiramente estável e relativizarem a dependência de características superficiais facilmente alteráveis e das atividades moldadas socialmente, se pode considerar o processo terminado. Este, não é independente do desenvolvimento cognitivo (Emmerich et al., 1977; Goldman e Goldman, 1982, Kohlberg, 1972, Lopez, 1982, McGonaghy, 1979, Slaby e Frey, 1975 e Thompson, 1975) estando em estreita relação com ele, existindo evidências de que o egocentrismo infantil retarda tais aquisições (Barragan, 1988) e de que as crianças se concebem a si próprias, como possuidoras de uma identidade sexual que não muda, pelos mesmos processos que concebem a identidade invariável dos objetos físicos, sendo a reversibilidade de pensamento, que lhes possibilita entender a invariância subjacente às transformações percetuais. Investigações relevantes na área da identidade sexual foram conduzidas por Bem (1989); Emmerich et al., (1977), Gordon et al., (1990); Goldman e Goldman (1982); Lopez (1984); Marcus e 
Overton (1978); McGonaghy (1980); Money e Ehrhardt (1982), Ruble et al., (2007); Slaby e Frey (1975); Thompson e Bentler (1973), sendo uma das mais controversas ideias na literatura, a de constância de género (Carey, 1985; Maccoby, 1999; Ruble et al, 2007). Na opinião de Carey (1985), o conceito de constância de género parece requerer necessariamente que as crianças compreendam a existência de uma "essência biológica subjacente" individual masculina ou feminina, que permanece invariante através das transformações superficiais. Muito acontece nas conceções infantis até as crianças verem o masculino e o feminino como categorias de base biológica (Carey, 1985; Volbert, 1996).

\section{Métodos}

Estabeleceram-se como objetivos desta investigação: conhecer o tipo de crenças infantis acerca de identidade sexual, assim como o processo de aquisição das mesmas e analisar as relações existentes entre o processo evolutivo e características de cada estádio das mesmas crenças e as variáveis sócio demográficas e de desenvolvimento. Trata-se de um estudo correlacional, quase experimental, com metodologia qualitativa e quantitativa, tendo sido o inquérito por entrevista e por questionário os principais métodos de recolha de dados para aferir as crenças das crianças nas categorias consideradas: conservação da identidade sexual verbalizada e conservação da identidade sexual em figuras com contradição (constância sexual). A nossa amostra é incidental, constituída por 566 crianças dos 3 aos 9 anos, selecionadas em pré-escolas e escolas do $1^{\circ}$ CEB da rede pública, no distrito e concelho da Guarda. Após pedido formal aos Agrupamentos de Escolas da Área Educativa da Guarda, para a realização do estudo, foram distribuidos 1000 questionários com o pedido de dados socio demográficos aos pais das crianças e realizadas as entrevistas semiestruturadas às crianças, nas quais utilizámos um método similar ao denominado método clínico de Piaget (Piaget, 1982). Todas as entrevistas começaram com as provas clássicas de conservação de sólidos e líquidos de Piaget (Piaget, 1967, 1978, 1981; Piaget e Inhelder, 1969; Sprinthall e Sprinthall, 1990). No tratamento estatístico dos dados, realizámos análise de frequências, percentagens, testes de Qui Quadrado e clusters. Em função dos objetivos, as variáveis contempladas apresentam-se em dois grupos gerais: independentes (sócio demográficas e de desenvolvimento) e dependentes (crenças infantis nos domínios analisados). Definimos três variáveis sócio demográficas: idade, sexo, nível sócio económico. Embora sem termos a pretensão de analisar a temática do desenvolvimento cognitivo, sentimos necessidade de incluir, uma variável relativa à classificação das crianças, em termos de conservação cognitiva entendida em termos piagetianos. Do registo de cada prova resultou a atribuição do nível de conservação cognitiva da criança em: 1. Não conservador (a mudança de forma plasticina ou água implica desigualdade); 2. Intermédio (às vezes admite igualdade e outras não) e 3 . Conservador (admite a igualdade com argumentos lógicos em todas situações).
Definimos como variáveis dependentes as crenças infantis de identidade sexual: conservação de identidade verbalizada; conservação de identidade em figuras com contradição (constância sexual). A variável conservação da identidade sexual verbalizada contem de forma sistematizada os resultados obtidos nas perguntas de conservação verbalizada realizadas: és rapaz ou rapariga? quando fores maior serás rapaz ou rapariga? e quando fores ainda maior serás papá ou mamã? e se te vestires de (o contrário à sua identidade) então... que serás? se quiseres quando fores maior podes ser... (o contrário à sua identidade sexual?). A variável conservação da identidade sexual em figuras com contradições (constância sexual) contem os resultados obtidos nas perguntas referentes a dois puzzles com erros: um com a figura de um homem nu com cara e cabelo de mulher e o outro com a figura de uma mulher nua com cara, cabelo e barba de homem. Todas as crenças são variáveis categoriais. Para todas as categorias considerámos o grau de elaboração e de coerência das respostas dadas, bem como a articulação dos diversos fatores de explicação. A categorização efetuada foi a seguinte: i) Conservação de Identidade Sexual Verbalizada. Na elaboração dos três níveis de resposta considerámos a resposta e os argumentos empregues nas perguntas de conservação verbalizada. No nível 1 (não conservador) a criança não conserva a sua a identidade sexual, considerando que ela depende da sua vontade, que muda ou que depende do papel. No nível 2 (intermédio) a criança encontra-se num estado intermédio, pois conserva a sua identidade sexual se não houver contradição. No nível 3 (conservador) conserva a identidade sexual, considerando que ela não depende da vontade, não muda nem depende do papel; ii) Conservação da Identidade Sexual em Figuras com Contradição (Constância Sexual). Na elaboração dos cinco níveis de resposta considerámos a descoberta das contradições, nos puzzles apresentados e os argumentos empregues: 1. Identidade por papel; 2. Identidade por papel, mas descobre o erro; 3. Identidade por genitais, mas descobre o erro (constância genital); 4. Não pode ser/ erro (constância genital); 5. Identidade por genitais relativizando o papel (constância genital). No nível 1, a criança não resiste às contradições expressas nas figuras, muitas vezes nem as notando, não conservando a identidade sexual das mesmas. No nível 2, a criança descobre o erro, mas define a identidade da figura, por características de papel, centrando-se no cabelo, barba ou cara. Nos níveis seguintes, a criança evolui, para graus de conservação de identidade superiores (aquisição de constância sexual) com resistência às contradições expressas nas figuras. No nível 3, a identidade é determinada pelos genitais, sendo a criança capaz de descobrir a contradição situando-a nas características de papel. No nível 4, a resistência à contradição aumenta e a criança reconhece que o puzzle não pode ser ou que está errado. O nível 5 é o mais evoluído, sendo a criança capaz de definir a identidade por genitais, relativizando o papel. 


\section{Resultados}

A distribuição das crianças por idade e sexo é uniforme. Em relação ao nível sócio económico, o nível baixo apresenta um peso que é o dobro dos níveis médio e alto. Através da análise de frequências e percentagens das várias categorias de respostas, e da análise estatística realizada, comprovámos a existência de percursos evolutivos ou de diferenciação nas várias crenças analisadas, sob a associação com diversos fatores. Apresentamos na tabela 1 as associações obtidas entre as crenças analisadas e as variáveis consideradas (idade; sexo; nível socio económico e nível de desenvolvimento cognitivo entendido em termos Piagetianos). Assinalamos com $\mathrm{x}$ as relações significativas comprovadas.

Tabela 1.

Sistematização de resultados

\begin{tabular}{lcccc}
\hline Variáveis Dependentes & \multicolumn{4}{c}{ Variáveis Independentes } \\
\hline & Idade & Sexo & Nse & NdcP \\
Conservação da & $\mathrm{X}$ & & $\mathrm{x}$ & $\mathrm{x}$ \\
$\begin{array}{l}\text { Identidade Sexual } \\
\text { verbalizada }\end{array}$ & & & & \\
Conservação da & $\mathrm{X}$ & $\mathrm{x}$ & $\mathrm{x}$ \\
Identidade Sexual em & & & & \\
Figuras com contradição & & & & \\
& & & & \\
\hline
\end{tabular}

A idade é determinante no processo de construção e elaboração das crenças consideradas, comprovando-se que acompanha o processo de evolução das crenças. $\mathrm{Na}$ variável da Conservação da Identidade Sexual Verbalizada, a percentagem de "não conservador" é superior no grupo dos 3 aos 4 anos, diminuindo acentuadamente, no grupo dos 5 e 6 anos, existindo apenas em percentagem baixa, no grupo dos 7 aos 9 anos. Estas crianças, identificam-se com o seu próprio sexo, dizendo que são rapazes ou raparigas e que vão ser com0o as mães ou como os pais, usando argumentos de papel, rótulos e características culturais. Consideram que a sua identidade pode mudar se quiserem, bastando vestirem roupa característica do sexo oposto, para a mudança de identidade acontecer, sendo reversível quando tirarem a roupa e voltarem a vestir a sua. Eu sou uma menina porque ando sempre com fitas no cabelo $e$ com mochilas de menina; vou ser sempre menina $e$ mulher porque vou ter mamas grandes como a mãe; vestida de rapaz sou menina porque me conhecem a cara; as meninas são sempre mães (6 anos, rapariga). A percentagem da categoria de "intermédio" é superior no grupo dos 5 aos 6 anos não existindo no grupo dos 3-4 anos, e existindo em percentagem baixa no grupo dos 7 aos 9 anos. Estas crianças conservam a sua identidade, desde que não exista contradição sugerida, sendo ainda suscetíveis a ela e não lhe resistindo de forma consistente. Eu sou rapaz porque nasci rapaz e só posso ser homem quando crescer porque tenho pilinha como o pai! vestido de menina fico menina com totós e saia; se quiser não posso ser mulher porque sou rapaz e só posso ser homem
(7 anos, rapaz). A categoria de "conservador" ocorre entre os 5 e os 9 anos, aumentando a sua percentagem com o aumento de idade. Neste período, as crianças, identificam-se com o seu próprio sexo, utilizando argumentos genitais e resistindo à contradição, afirmando de forma convincente e determinada, que os genitais são determinantes e que a identidade não muda com a roupa ou com o papel. Sou menina porque sou do sexo feminino e se me vestir de rapaz não mudo de sexo, é claro porque ninguém muda de sexo; vou ser mulher e nunca posso ser homem porque o sexo com que nascemos é para sempre (8 anos, rapariga). Comprovámos, assim, que a progressão na aquisição da conservação da identidade sexual verbalizada evolui da não conservação da identidade sexual (100\% no grupo dos 3-4 anos), até à sua conservação ( $94.7 \%$ no grupo dos 7-9 anos), passando por um período intermediário (30.4\%, no grupo dos 5-6 anos). Das crianças entrevistadas nenhuma realizou o processo inverso. Relativamente à variável Conservação da Identidade Sexual em Figuras com Contradição (Constância Sexual), a "identidade por papel" ocorre dos 3 aos 6 anos, diminuindo a sua percentagem com o aumento da idade. É um homem porque tem barbas, esta é uma mulher porque tem cabelo de mulher (5 anos, rapaz). A mesma identidade, mas com descoberta de erro, apresenta percentagem superior no grupo dos 5- 6 anos: estes desenhos não estão bem; este homem (porque tem barbas) não pode ter maminhas nem pipi e esta mulher (caracóis) não pode ter pilinha porque assim não dá certo; os homens têm pilinha e as mulheres pipi (4 anos, rapariga). A "identidade por genitais, com descoberta do erro" (aquisição de constância sexual), ocorre dos 5 aos 9 anos, aumentando a sua percentagem para as idades superiores. Esta é mulher porque tem bexiga e mamas mas tem a cabeça errada porque as mulheres não têm barba; este é um homem porque tem pénis, mas esta cabeça de senhora está mal (9 anos, rapariga). As categorias de resposta "não pode ser / erro" e "identidade por genitais relativizando o papel", ocorrem apenas no grupo dos 7-9 anos: Isto é impossível! Ninguém tem corpo de mulher e cara de homem e ninguém tem corpo de homem e cara de mulher; só mesmo nestes bonecos (8 anos, rapaz). Os resultados obtidos, comprovam que, a conservação da identidade sexual em figuras com contradição (aquisição de constância sexual) é mais tardia do que a conservação verbalizada tendo sido comprovado que muitas crianças resistem já à contradição verbalizada, não resistindo, contudo, momentos a seguir, à contradição expressa nos puzzles, e só posteriormente afirmam não poder existir a situação caricaturada na figura, de um corpo de mulher com cabeça de homem ou vice-versa. Comprovámos, pois, que na conservação da identidade sexual em figuras com contradição, existe um processo evolutivo (que acompanha a idade), da ausência de conservação (presente nas categorias de identidade de papel sem descoberta de erro e identidade de papel com descoberta de erro), à conservação progressiva, da constância genital (presente nas categorias de identidade por genitais com descoberta de erro, afirmação da impossibilidade da contradição e identidade por genitais com relativização do papel). Na generalidade, as crenças infantis, não estão 
relacionadas com o sexo. As crenças infantis consideradas estão relacionadas com o nível sócio económico, correspondendo aos níveis sócio económicos mais elevados, crenças mais evoluídas. As crianças conservadoras a nível de quantidades contínuas e descontínuas (relativamente ao nível de desenvolvimento cognitivo em termos piagetianos) têm crenças mais evoluídas do que as crianças não conservadoras. A percentagem de não conservadores, relativamente à permanência de conservação de identidade sexual, é superior para os não conservadores, enquanto a percentagem de conservadores é superior para os intermédios e conservadores. No âmbito da conservação da identidade sexual em figuras com contradição, a percentagem de "identidade por papel" é superior para os não conservadores, a "identidade por papel com descoberta do erro"; diminui para os intermédios e não existe nos conservadores. A percentagem de "identidade por genitais, mas descobre o erro" (constância genital), aumenta muito dos não conservadores para os intermédios, continuando a aumentar para os conservadores. A categoria de "não pode ser - erro" é superior para os conservadores e a "identidade por genitais, relativizando o papel" existe apenas nos conservadores.

\section{Discussão}

O nosso estudo revelou que as crianças do grupo de 34 anos, não conservam a sua identidade nas provas de conservação verbalizada $(100 \%)$; não resistem à contradição expressa em figuras, definindo a identidade sexual das mesmas, com argumentos de papel (70.2\%), descobrindo por vezes o erro que situam nos genitais (29.8\%). No grupo de 5-6 anos, as crianças, não conservam a sua identidade nas provas de conservação verbalizada (47.8\%), começando, no entanto, a transitar para o período de conservação intermédia $(30.4 \%)$ ou mesmo de conservação $(21.7 \%)$. Não resistem à contradição expressa em figuras, definindo a identidade sexual das mesmas, com argumentos de papel, onde descobrem vulgarmente o erro, que situam nos genitais (65.8\%), começando a evoluir para uma fase em que definem a identidade por genitais e situam o erro na cabeça ou no papel (11.8\%). As crianças do grupo de 79 anos conservam a sua identidade, nas provas de conservação verbalizada $(94.7 \%)$; conservam a identidade sexual das figuras com contradição que definem pelos genitais, descobrindo o erro, que situam na cabeça $(68 \%)$ ou afirmando que não pode ser $(21.7 \%)$. Em relação à conservação da identidade sexual, verificámos que é adquirida na generalidade, por algumas crianças, com pouca resistência à sugestão contrária, entre os 5 e os 6 anos, sendo apenas entre os 6 e os 8 anos, que é adquirida com nítida resistência à sugestão contrária (Bem, 1981; Emmerich et al., 1977, Gordon et al., 1990; Goldman e Goldman 1982; Lopez, 1984; Marcus e Overton, 1978; McGonaghy, 1979; Money e Ehrhardt, 1982, Ruble et al. 2007; Slaby e Frey, 1975; Thompson e Bentler, 1973). Nas figuras com contradições (cabeça de mulher com corpo de homem e cabeça de homem com corpo de mulher), todas as crianças até aos 6 anos fizeram uma descriminação pela cabeça e só posteriormente identificaram as contradições, adquirindo apenas nesta fase a verdadeira noção de constância genital. Só entre os 7 e 9 anos, além de reconhecerem o erro, afirmaram que eram os genitais o determinante, tal como defendem outras investigações (Kohlberg, 1972; Emmerich et al, 1977; Marcus e Overton, 1978; Lopez, 1984). Comprovámos a existência de relações significativas, entre o nível de desenvolvimento cognitivo, o processo de construção da identidade sexual e a aquisição de constância sexual. As crianças não conservadoras, em termos de desenvolvimento cognitivo, entendido em termos piagetianos, diferenciam-se significativamente das crianças intermédias e conservadoras, na elaboração do processo mencionado. Comprovámos assim, os estudos de Emmerich et al. (1977), Goldman e Goldman (1982), Kohlberg (1972), Lopez (1988), McGonaghy (1980), Slaby e Frey (1975) e Thompson, (1973), que encontraram uma progressão desenvolvimental, na compreensão das crianças acerca de identidade sexual e de género, relacionada com os conceitos piagetianos de desenvolvimento cognitivo. Consideramos que na base desta relação, está a capacidade de diferenciar, identificar e explicar, com explicações genitais, que é superior nos intermédios e conservadores, sendo o maior conhecimento da diferenciação genital, que implica que a percentagem de conservadores de identidade sexual verbalizada seja superior nos grupos de intermédios e conservadores em desenvolvimento cognitivo. Pensamos poder apoiar-nos, neste ponto, na opinião de Carey (1985) ao considerar que, ver o género como uma parte da essência biológica de cada pessoa, impulsiona de facto o caminho para a aquisição da sua constância e ainda na opinião de Bem (1981), segundo a qual, o conhecimento das crianças sobre a diferenciação genital está relacionado positivamente, com o processo de aquisição de constância da mesma. A este respeito, verificámos que no grupo de não conservadores cognitivos, existe predominância do nível de não conservação de identidade sexual, existindo, no entanto, alguns intermédios (14.3\%) e conservadores (16.3\%) de identidade, neste grupo. Pensamos que este facto significa que, embora os dois processos estejam relacionados, existe uma anterioridade temporal ligeira na conservação de identidade sexual face à conservação cognitiva. A capacidade de constância sexual, com resistência dupla à contradição (verbal, face às contradições sugeridas e visual, perante as contradições observadas nos puzzles), aumenta muito, do grupo de não conservadores em nível cognitivo (12\%), para os intermédios $(79.2 \%)$, continuando a aumentar, para os conservadores (100\%). Este facto reforça a explicação da anterioridade referida, pois sem ela, esperaríamos que o nível de constância sexual (pelas contradições a que está exposto nos testes) existisse apenas no grupo de conservadores cognitivos, o que não acontece. O presente estudo, comprova a importância do nível sócio económico, no processo de construção de identidade sexual. Tal como referimos, a evolução do estatuto sócio económico acompanha o processo de elaboração das crenças consideradas. Partilhamos a opinião de Gordon 
et al., (1990) acerca da influencia da classe social, como sendo resultado de atitudes mais restritivas dos pais, de classe social baixa, quanto à educação sexual dos seus filhos.

\section{Conclusões}

Trata-se de um estudo com uma amostra incidental, pelo que não pode ser generalizado. Em primeiro lugar comprovámos a existência de processos evolutivos ou de diferenciação nas crenças infantis analisadas, associados a vários fatores. Em segundo lugar comprovámos que, além da idade, o nível sócio económico e o nível de desenvolvimento cognitivo (entendido em termos piagetianos), estão associados, significativamente, às características específicas relativas ao grau de elaboração das crenças infantis. Em terceiro lugar verificámos a existência de padrões evolutivos significativamente semelhantes nas crenças das crianças pertencentes aos dois sexos, embora com tendência para que as crenças das raparigas sejam mais evoluídas que as dos rapazes. Em último lugar gostaríamos de sublinhar a importância das características da personalidade infantil (por exemplo, curiosidade e extroversão) na construção (enquanto apreensão e elaboração) do conhecimento, fator chave e explicativo das diferenças manifestadas, em crianças do mesmo grupo de idades. Sublinhamos, finalmente, a matriz de fatores biológicos, sociais, cognitivos, motivacionais e educacionais em que se alicerça a construção do seu conhecimento.

\section{Referências}

Atkinson, R.L., Atkinson, R.C., Smith. E.E. e Bem, D.J. (1995). Introdução à Psicologia. Tradução, Dayse Batista. Artes Médicas: Porto Alegre.

Barragán, F. (1988). Las Teorías Sexuales Infantiles, la Información Sexual y las teorías implícitas de los adultos sobre la sexualidad y educación sexual: Bases para el diseño curricular de la educación sexual en el ciclo medio de la Egb. Tesis Doctoral, Universidad De La Laguna.

Barragàn, F. (1991). La Educacion Sexual. Guia Teórica y Práctica. Paidos: Barcelona.

Badinter, E. (1992). XY A Identidade Masculina. Edições ASA: Porto.

Bem, S. L. (1981). Gender Schema Theory: A Cognitive Account Of Sex-Typing. Psychological Review, 88, 354-364.

Bernstein, A. (1994). Flight of the Stork: What Children Think (And When About Sex and Family Building). Perspectives. Revised edition: Indianapolis.

Bussey, K. E Bandura, A. (1999). Social Cognitive Theory Of Gender Development And Differentiation. Psychological Review, 106, 676-713.

Calderone, M. e Johnson, E. (1983). The Family Book About Sexualiy. Bantam Books: New York.

Carey, S. (1985). Conceptual Change In Childhood. Mit Press: Cambridge.

Emmerich, Goldman, Kirsh e Sharabany (1977). Evidence For a Transitional Phase, in the Development of Gender Constancy. Child Development, 48, 930936.

Gesell, A. (1956). Psicología Evolutiva de 1 a 16 años. Paidós. Buenos Aires.

Goldman, R. e Goldman, J. (1982). Children's Sexual Thinking. Routledge And Kegan Paul: London.

Gordon, B.; Schroeder, C. E Abrams, J. (1990). Age And Social Class Differences. In Children's Knowledge Of Sexuality. Journal Of Clinical Child Psychology, 19, 33-43.

Kohlberg, L. (1972). Análisis de los conceptos y actitudes infantiles relativas al papel sexual desde el punto de vista y del desarrollo cognitivo. IN Desarrollo de las diferencias sexuales. Marova: Madrid.

López, F. (1988). La Adquisición De La Identidad Sexual $Y$ De Género: I. Infancia- Adolescencia. In Fernandez, J. Nuevas Perspectivas Del Sexo E Del Genero. Pirámide: Madrid.

López, F. e Fuertes, A. (1989). Para Compreender a Sexualidade. APF: Lisboa.

López, F. (2005). La Educación Sexual. Editorial Biblioteca Nueva: Madrid.

Maccoby, E. (1999). The Two Sexes. Growing up apart, Coming Together. Harvard University Press. Cambridge.

Mantilla, L. (2006). Rapazes, Raparigas: Iguais, Mas Diferentes. In J. Bas et al (Eds.), Enciclopédia Dos Pais. Como Ser Melhores Pais. Parte I Enciclopédia Dos Pais. Como Ser Melhores Pais, Parte I, (Pp. 98101). Imprensa Livre, S. A: Rio De Mouro.

Marcus, D. E., E Overton, W. F. (1978). The Development Of Cognitive Gender Constancy And Sex Role Preferences. Child Development, 49, 434444.

Marques, A. M. ; Vilar, M. e Forreta, F. (2002). Os Afectos e a Sexualidade na Educação Pré-Escolar. Texto Editora: Lisboa.

Mcconaghy, M. J. (1980). The Gender Understanding Of Swedish Children. Child Psychiatry And Human Development, 11, 19-32.

Money, J. e Ehrhardt, A. (1982). Desarrollo de la sexualidad humana (Diferenciación y dimorfismo de la identidad de género). Morata, Madrid.

Piaget, J. (1981). La Representación del Mundo en el niño: Morata, Madrid.

Piaget, J., e Inhelder, B. (1969). The psychology of the child. Basic: New York.

Ruble, D. N., Taylor, L., Cyphers, L., Greulich, F., Lurye, L. E Shrout, P. (2007). The Role Of Gender Constancy In Early Gender Development. New York University. Child Development, Volume 78, Number 4, Pages $1121-1136$.

Slaby, R. G. e Frey, K. S. (1975). Development Of Gender Constancy And Selective Attention To SameSex Models. Child Development, 46, 849 - 856.

Volbert, R. (1996). Sexual Knowledge of Preschool Children. In Sandfort, T. e Rademakers, J. (Eds), Childhood Sexuality: Normal Sexual Behavior and Development. The Haworth Press, Inc., New York, USA. 